\title{
Identification of hub genes and pathways in glioblastoma by bioinformatics analysis
}

\author{
SHOUBO YANG ${ }^{1 *}$, KAIDI GAO $^{2 *}$ and WENBIN LI ${ }^{1}$ \\ ${ }^{1}$ Department of Neuro-Oncology, Neurosurgery Center, Beijing Tiantan Hospital, Capital Medical University, Beijing 100050; \\ ${ }^{2}$ Continuing Education and Training Department, Beijing Rehabilitation Hospital Affiliated to Capital Medical University, \\ Beijing 100144, P.R. China
}

Received February 9, 2018; Accepted September 11, 2018

DOI: $10.3892 / \mathrm{ol} .2018 .9644$

\begin{abstract}
Glioblastoma (GBM) is the most common type of malignant brain tumor, and is associated with poor patient prognosis. A comprehensive understanding of the molecular mechanism underlying GBM may help to guide the identification of novel diagnoses and treatment targets. The gene expression profile of the GSE4290 GBM dataset was analyzed in order to identify differentially expressed genes (DEGs). Enriched pathways were identified through Gene Ontology and the Kyoto Encyclopedia of Genes and Genomes analyses. A protein-protein interaction network was constructed in order to identify hub genes and for module analysis. Expression and survival analyses were conducted in order to screen and validate critical genes. A total of 1,801 DEGs were recorded, including 620 upregulated and 1,181 downregulated genes. Upregulated DEGs were enriched in the terms 'mitotic cell cycle process', 'mitotic cell cycle' and 'cell cycle process'. Downregulated genes were enriched in 'transsynaptic signaling', 'anterograde transsynaptic signaling' and 'synaptic signaling'. A total of 15 hub genes, which displayed a high degree of connectivity, were selected. These genes included vascular endothelial growth factor A, cyclin-dependent kinase 1 (CDK1), cell-division cycle protein 20 (CDC20), aurora kinase A (AURKA), and budding uninhibited by benzimidazoles 1 (BUB1). The identified DEGs and hub genes may help guide investigations on the mechanisms underlying the development and progression of GBM. CDK1, CDC20, AURKA and BUB1, which are involved in cell cycle pathways, may be potential targets in the diagnosis and therapy of GBM.
\end{abstract}

Correspondence to: Dr Wenbin Li, Department of Neuro-Oncology, Neurosurgery Center, Beijing Tiantan Hospital, Capital Medical University, 6 Beijing Tiantan Xili, Beijing 100050, P.R. China

E-mail: liwenbin@ccmu.edu.cn

*Contributed equally

Key words: glioblastoma, bioinformatics analysis, protein-protein interaction, pathway analysis, module analysis

\section{Introduction}

Glioblastoma (GBM) is the most fatal primary malignant tumor of the central nervous system in adults, and accounts for $46.1 \%$ of all cases of malignant brain tumors (1). Patients who are newly diagnosed with GBM receive surgery as standard, followed by concurrent radiochemotherapy and maintenance temozolomide chemotherapy (2). Despite this aggressive treatment strategy, relapse is common and the median overall survival (OS) of patients with GBM is $\sim 15$ months (3). No other effective agents against GBM have been developed over the past decade since the approval of temozolomide for GBM treatment in 2004. Furthermore, the use of currently available agents has been hindered due to limited information on the molecular mechanisms involved in GBM development or treatment response. Therefore, it may be beneficial to elucidate the mechanisms underlying GBM and consequently develop novel therapeutic strategies.

Numerous studies have investigated the genes involved in GBM. A previous study by Yeom et al (4) indicated that the expression of the guanosine-5'-triphosphate-binding protein Ras related glycolysis inhibitor and calcium channel regulator is correlated with temozolomide resistance, and contributes to the poor survival of patients with GBM. The inhibitor of nuclear factor $\kappa$-B kinase subunit $\varepsilon$ (IKBKE) is overexpressed in human GBM, and the inhibition of IKBKE markedly suppresses the proliferative and invasive activity of GBM cells (5). High expression levels of hypoxia-inducible factor- $1 \alpha$ promote the activation of glioma cell motility by affecting molecules associated with invasion (6). Recombinant expression of HMG-CoA reductase (HMGCR) promotes the growth and migration of U251 and U373 cells, whereas the knockdown of HMGCR expression inhibits the growth, migration and metastasis of GBM cells (7). Lymphoid enhancer factor-1 maintains the state of proliferation and migration in GBM cells, and the GBM stem-cell-like self-renewal ability of U251 cells (8). However, the current understanding of the mechanisms underlying GBM remains limited.

In 2006, Sun et al (9) published a study in which 157 primary human glioma and 23 nontumor human brain samples underwent mRNA expression profiling, in order to verify whether overexpression of stem cell factors was associated with the poor prognosis of patients with glioma. In the 
current study, microarray analysis was conducted to screen differentially expressed genes (DEGs) in GBM samples. Hub genes, in addition to significant modules and pathways, were identified using comprehensive bioinformatics methods. The present study aimed to identify the candidate genes and associated pathways of GBM, in order to elucidate the molecular mechanisms underlying this malignancy.

\section{Materials and methods}

Microarray data. The gene expression profiles of GSE4290 were downloaded from the public functional genomics data repository Gene Expression Omnibus (GEO; http://www.ncbi. nlm.nih.gov/geo/), which is based on the Affymetrix (Thermo Fisher Scientific, Inc., Waltham, MA, US) Human Genome U133 Plus 2.0 Array. These gene expression files were deposited by Sun et al (9). The gene expression profiles of 77 GBM tissue samples and 23 nontumor brain samples from patients with epilepsy were retrieved from the GSE4290 dataset.

DEG screening. GEO2R is an interactive online tool based on the $\mathrm{R}$ programming language, which allows for comparisons between two groups of samples in a GEO series to be made (10). Adjusted P-values were utilized to decrease the false-positive rate through the default Benjamini and Hochberg false discovery rate method. An adjusted $\mathrm{P}<0.05$ and $\mid \log \mathrm{FCl} \geq 2$ were considered to indicate a statistically significant difference.

Functional enrichment analysis. Gene Ontology (GO) analysis may be applied in large-scale functional studies on genomic or transcriptomic data (11). The Kyoto Encyclopedia of Genes and Genomes (KEGG) is the major recognized pathway-associated database, which contains information on gene networks in various organisms (12). Previous studies have claimed that the analysis of upregulated and downregulated genes separately may allow for the identification of additional pathways, compared with combined analysis (13-15). In the present study, specific pathways involved in tumor occurrence and development were used; hence, separate analysis was performed. GO functional and KEGG pathway enrichment analyses were conducted separately for upregulated and downregulated genes using the Database for Annotation, Visualization, and Integrated Discovery software (DAVID version 6.8; http://david.ncifcrf.gov/) (16). $\mathrm{P}<0.05$ was considered to indicate a statistically significant difference.

Integration of protein-protein interaction (PPI) network and module analysis. The STRING (https://string-db.org/) database is an online tool for the assessment and integration of PPIs, including direct (physical) and indirect (functional) associations. STRING version 10.5 encompasses 9,643,763 proteins from 2,031 organisms (17). PPI associations amongst DEGs were searched for using the STRING database with a default required confidence of $>0.4$. The PPI networks of the DEGs were constructed using Cytoscape software version 3.6.0 (http://www.cytoscape.org/). The plug-in Molecular Complex Detection (MCODE) was used to screen important modules with established scores of $>3$ and nodes of $>4$. GO and KEGG analyses were also conducted using the genes in these modules. In the PPI network, the number of edges involved determined the degrees of the nodes, and nodes with high degrees were determined to be hub genes. Hub genes were also mapped to STRING in order to evaluate their PPI information.

Expression and survival analyses of hub genes. Gene Expression Profiling Interactive Analysis (GEPIA) is an online tool used to analyze the RNA sequencing expression data of 9,736 tumors and 8,587 healthy samples from The Cancer Genome Atlas (TCGA) and the Genotype-Tissue Expression (GTEx) databases (18). GEPIA was used to perform the tumor/healthy differential expression and survival analyses of hub genes. The method of Kaplan-Meier for survival analysis was conducted in GEPIA between the high and low expression groups, with a cut-off value of 50\%. The hazard ratio with $95 \%$ confidence intervals and the log-rank P-value were calculated, and the results are displayed as a plot. $\mathrm{P}<0.05$ was considered to indicate a statistically significant difference.

\section{Results}

Identification of DEGs. The comparative GEO2R analysis of the DEGs in the GBM samples and healthy controls revealed 1,801 DEGs, including 620 upregulated and 1,181 downregulated genes.

GO function and KEGG pathway enrichment analysis. The upregulated and downregulated DEGs were imported into DAVID for GO analysis. The GO analysis results revealed that the upregulated DEGs were significantly enriched in the terms 'mitotic cell cycle process', 'mitotic cell cycle' and 'cell cycle process' (Table I). Downregulated genes were enriched in 'trans-synaptic signaling', 'anterograde trans-synaptic signaling' and 'synaptic signaling' (Table I).

The most significantly enriched KEGG pathways of the upregulated and downregulated DEGs are displayed in Table II. The upregulated DEGs were enriched in 'cell cycle', 'ECM-receptor interaction', 'PI3K-Akt signaling pathway, 'p53 signaling pathway' and 'focal adhesion'. Downregulated DEGs were enriched in 'morphine addiction', 'GABAergic synapse', 'retrograde endocannabinoid signaling', 'calcium signaling pathway' and 'glutamatergic synapse'.

PPI network and module analyses. The PPI network constructed for the DEGs had 993 nodes and 7,810 interactions, in which two of the most significant modules were identified by MCODE (Fig. 1). Module 1 had 59 nodes and 1,576 interactions, whereas Module 2 had 32 nodes and 496 interactions. DEGs in these modules were also enriched in 'cell cycle' and 'neuroactive ligand-receptor interaction' of the KEGG pathways (Fig. 1). The top 15 hub genes were selected by the PPI network, with a degree of $>81$ (Fig. 2).

Expression level and Kaplan-Meier plot of hub genes. The expression levels of all 15 hub genes in patients with GBM were upregulated relative to those in the healthy controls $(\mathrm{P}<0.05)$. High expression levels of vascular endothelial growth factor A (VEGFA) was associated with poor prognosis of patients with GBMs, whereas no significant difference was 
Table I. GO analysis of differentially expressed genes associated with glioblastoma.

A, upregulated genes

\begin{tabular}{|c|c|c|c|c|}
\hline Term & Count & $\%$ & P-value & FDR \\
\hline GO:1903047: Mitotic cell cycle process & 80 & 17.699 & $9.95 \times 10^{-25}$ & $1.90 \times 10^{-21}$ \\
\hline GO:0000278: Mitotic cell cycle & 83 & 18.362 & $2.43 \times 10^{-24}$ & $4.64 \times 10^{-21}$ \\
\hline GO:0022402: Cell cycle process & 98 & 21.681 & $2.27 \times 10^{-23}$ & $4.35 \times 10^{-20}$ \\
\hline GO:0007049: Cell cycle & 107 & 23.672 & $1.40 \times 10^{-21}$ & $2.68 \times 10^{-18}$ \\
\hline GO:0051301: Cell division & 59 & 13.053 & $7.57 \times 10^{-21}$ & $1.45 \times 10^{-17}$ \\
\hline GO:0007067: Mitotic nuclear division & 48 & 10.619 & $2.22 \times 10^{-18}$ & $4.25 \times 10^{-15}$ \\
\hline GO:0044770: Cell cycle phase transition & 54 & 11.946 & $2.47 \times 10^{-18}$ & $4.72 \times 10^{-15}$ \\
\hline GO:0044772: Mitotic cell cycle phase transition & 52 & 11.504 & $4.17 \times 10^{-18}$ & $7.96 \times 10^{-15}$ \\
\hline GO:0000819: Sister chromatid segregation & 35 & 7.743 & $5.93 \times 10^{-18}$ & $1.13 \times 10^{-14}$ \\
\hline GO:0000280: Nuclear division & 52 & 11.504 & $9.96 \times 10^{-16}$ & $1.91 \times 10^{-12}$ \\
\hline
\end{tabular}

B, downregulated genes

\begin{tabular}{|c|c|c|c|c|}
\hline Term & Count & $\%$ & P-value & FDR \\
\hline GO:0099537: Trans-synaptic signaling & 137 & 16.707 & $1.13 \times 10^{-64}$ & $2.15 \times 10^{-61}$ \\
\hline GO:0098916: Anterograde trans-synaptic signaling & 137 & 16.707 & $1.13 \times 10^{-64}$ & $2.15 \times 10^{-61}$ \\
\hline GO:0099536: Synaptic signaling & 137 & 16.707 & $1.13 \times 10^{-64}$ & $2.15 \times 10^{-61}$ \\
\hline GO:0007268: Chemical synaptic transmission & 137 & 16.707 & $1.13 \times 10^{-64}$ & $2.15 \times 10^{-61}$ \\
\hline GO:0007399: Nervous system development & 227 & 27.682 & $4.23 \times 10^{-45}$ & $8.05 \times 10^{-42}$ \\
\hline GO:0007267: Cell-cell signaling & 178 & 21.707 & $9.70 \times 10^{-41}$ & $1.85 \times 10^{-37}$ \\
\hline GO:0050804: Modulation of synaptic transmission & 66 & 8.048 & $1.69 \times 10^{-31}$ & $3.21 \times 10^{-28}$ \\
\hline GO:0048666: Neuron development & 124 & 15.121 & $2.61 \times 10^{-31}$ & $4.98 \times 10^{-28}$ \\
\hline GO:0031175: Neuron projection development & 111 & 13.536 & $5.22 \times 10^{-30}$ & $9.94 \times 10^{-27}$ \\
\hline GO:0007610: Behavior & 90 & 10.975 & $9.43 \times 10^{-30}$ & $1.80 \times 10^{-26}$ \\
\hline
\end{tabular}

FDR, false discovery rate; GO, gene ontology.

A

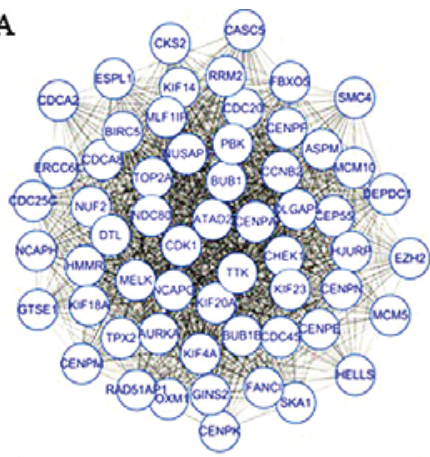

$\mathrm{C}$

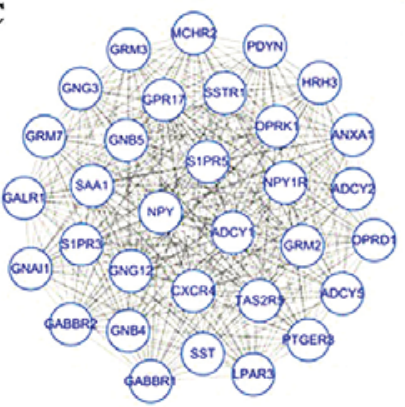

\begin{tabular}{|c|c|c|}
\hline Term & PValue & Genes \\
\hline Cell cycle & $3.81 \times 10^{-13}$ & $\begin{array}{l}\text { CDK1, CDC45, CCNB2, BUB1, TTK, } \\
\text { BUB1B, CHEK1, CDC20, ESPL1, } \\
\text { CDC25C, MCMS }\end{array}$ \\
\hline Oocytemeiosis & $4.35 \times 10^{-7}$ & $\begin{array}{l}\text { CDK1,BUB1,FBXO5,AURKA, } \\
\text { CDC20,ESPL1,CDC25C }\end{array}$ \\
\hline $\begin{array}{l}\text { p53 signaling } \\
\text { pathway }\end{array}$ & $3.48 \times 10^{-3}$ & CDK1, CCNB2, RRM2, CHEK1, GTSE1 \\
\hline
\end{tabular}

D

\begin{tabular}{lll}
\hline Term & PValue & Genes \\
\hline $\begin{array}{l}\text { Neuroactive } \\
\text { ligand- }\end{array}$ & $1.79 \times 10^{-14}$ & MCHR2, PTGER3, OPRK1, GABBRl, LPAR3, \\
receptor & & GABBR2, NPY1R, S1PR3, GRM3, GRM2, HRH, \\
interaction & & GALR1, SSTR1, GRM7, S1PRS, OPRD1 \\
Glutamaterge & $1.03 \times 10^{-11}$ & ADCY1, GRM3, ADCY2, GRM2, GNAIl, \\
synapse & & ADCY5, GRM7, GNB5, GNB4, GNG3, GNG12 \\
GABAergic & $2.39 \times 10^{-11}$ & ADCY1, ADCY2, GNAI1, ADCY5, GABBR1, \\
synapse & & GNB5, GNB4, GABBR2, GNG3, GNG12 \\
Morphine & $4.48 \times 10^{-11}$ & ADCY1, ADCY2, GNAIl, ADCY5, GABBR1, \\
addiction & & GNB5, GNB4, GABBR2, GNG3, GNG12 \\
\hline
\end{tabular}

Figure 1. Top two modules from the Protein-protein interaction network. (A) Module 1. (B) The enriched pathways of module 1. (C) Module 2. (D) The enriched pathways of module 2 . 
Table II. KEGG pathway analysis of differentially expressed genes associated with glioblastoma.

A, upregulated genes

\begin{tabular}{lccc}
\hline Term & Count & $\%$ & P-value \\
\hline hsa04110: Cell cycle & 19 & 4.203 & $7.83 \times 10^{-10}$ \\
hsa04512: ECM-receptor interaction & 16 & 3.539 & $1.89 \times 10^{-09}$ \\
hsa04151: PI3K-Akt signaling pathway & 28 & 6.194 & $5.08 \times 10^{-08}$ \\
hsa04115: p53 signaling pathway & 13 & 2.876 & $5.41 \times 10^{-08}$ \\
hsa04510: Focal adhesion & 19 & 4.203 & $2.20 \times 10^{-06}$ \\
hsa05205: Proteoglycans in cancer & 17 & 3.761 & $2.54 \times 10^{-05}$ \\
hsa04610: Complement and coagulation cascades & 10 & 2.212 & $3.88 \times 10^{-05}$ \\
hsa05150: Staphylococcus aureus infection & 9 & 1.991 & $4.15 \times 10^{-05}$ \\
hsa05166: HTLV-I infection & 18 & 3.982 & $1.49 \times 10^{-04}$ \\
\hline
\end{tabular}

$\mathrm{B}$, downregulated genes

\begin{tabular}{llll}
\hline Term & Count & $\%$ & P-value \\
\hline hsa05032: Morphine addiction & 32 & 3.902 & $5.34 \times 10^{-21}$ \\
hsa04727: GABAergic synapse & 30 & 3.658 & $9.98 \times 10^{-20}$ \\
hsa04723: Retrograde endocannabinoid signaling & 31 & 3.780 & $1.97 \times 10^{-18}$ \\
hsa04020: Calcium signaling pathway & 39 & 4.756 & $1.40 \times 10^{-17}$ \\
hsa04724: Glutamatergic synapse & 30 & 3.658 & $8.56 \times 10^{-16}$ \\
hsa05033: Nicotine addiction & 19 & 2.317 & $3.78 \times 10^{-15}$ \\
hsa04080: Neuroactive ligand-receptor interaction & 44 & 5.365 & $1.82 \times 10^{-14}$ \\
hsa04024: cAMP signaling pathway & 35 & 4.268 & $6.72 \times 10^{-13}$ \\
hsa04713: Circadian entrainment & 24 & 2.926 & $2.85 \times 10^{-12}$ \\
hsa05031: Amphetamine addiction & 19 & 2.317 & $8.49 \times 10^{-11}$ \\
hsa05032: Morphine addiction & 32 & 3.902 & $5.34 \times 10^{-21}$ \\
\hline
\end{tabular}

KEGG, The Kyoto Encyclopedia of Genes and Genomes; ECM, extracellular matrix; PI3K, phosphoinositide 3-kinase; HTLV, human T-cell leukemia-lymphoma virus; GABA, gamma-aminobutyric acid; cAMP, Cyclic adenosine monophosphate.

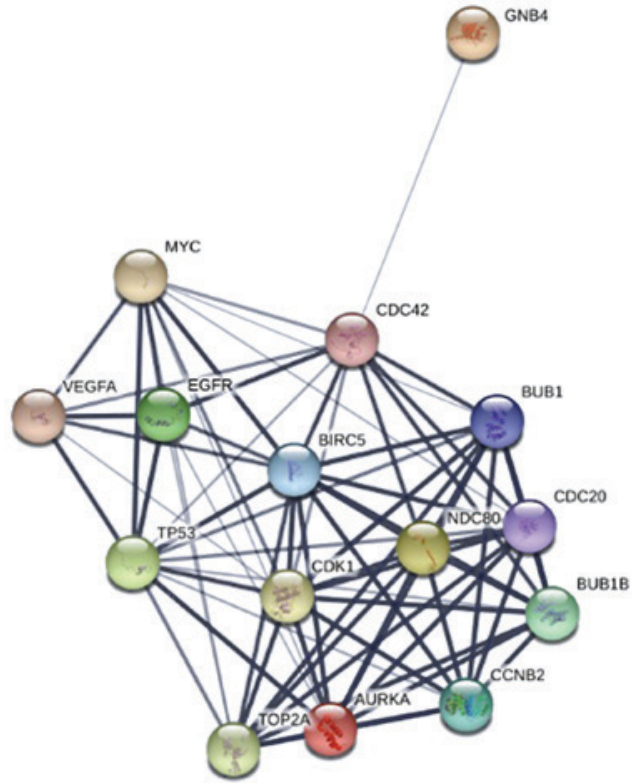

Figure 2. Protein-protein interaction network for the top 15 hub genes. Circles represent hub genes, and connecting lines between them represent interactions. observed for the remaining 14 genes (Fig. 3). The hub genes cyclin-dependent kinase 1 (CDK1), cell-division cycle protein 20 (CDC20), aurora kinase A (AURKA), and budding uninhibited by benzimidazoles 1 (BUB1) were also enriched in the top three modules.

\section{Discussion}

GBM is the most common primary malignant tumor of the brain. However, the molecular mechanisms underlying the progression of GBM remains unclear. In the present study, DEGs between GBM and healthy samples were identified, and a series of bioinformatics analytical methods applied in order to determine the key genes and pathways associated with GBM. A total of 1,801 DEGs were identified. These DEGs included 620 upregulated and 1,181 downregulated genes. Subjecting the DEGs to bioinformatics analysis, including GO enrichment, KEGG pathway, PPI network and survival analyses, revealed that GBM-associated genes and pathways may serve an important role in the initiation and progression of cancer.

GO term enrichment analysis indicated that the upregulated DEGs were significantly enriched in the terms "mitotic 
A

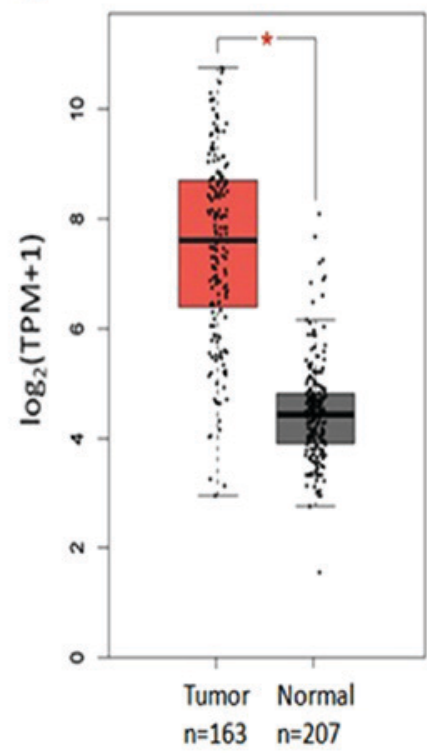

B

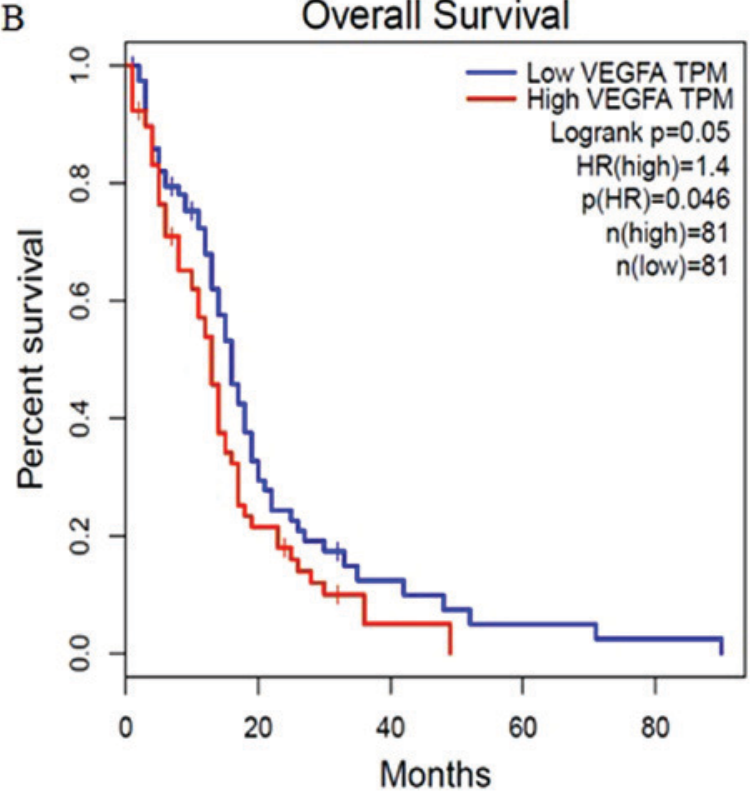

Figure 3. Expression and survival analysis of VEGFA. (A) Expression levels of VEGFA in GBMs compared with healthy controls. *P<0.05. (B) Survival analysis of high and low VEGFA expression levels in patients with GBM. VEGFA, vascular endothelial growth factor A; GBM, glioblastoma; HR, hazard ratio; TPM, transcripts per million.

cell cycle process', 'mitotic cell cycle' and 'cell cycle process'. Deregulation of the cell cycle serves a critical role in the proliferation of malignant glioma cells. Genetic analyses of primary human brain tumors detected common mutations in genes encoding proteins critical for cell cycle regulation. These genes include retinoblastoma protein, INK4A and CDK4 (19-22). The downregulated DEGs were enriched in pathways involved in trans-synaptic signaling and synaptic signaling. Yu et al (23) reported that metabotropic glutamate receptors, which are involved in synaptic signaling, are also involved in the transformation and maintenance of various cancer types, including glioma, melanoma skin cancer, breast cancer and prostate cancer. The WW and C2 domain-containing protein (WWC) family serves important roles in regulating cell proliferation, cell migration and synaptic signaling. The overexpression of WWC3 inhibits glioma cell proliferation, migration and invasion (24).

KEGG pathway analysis indicated that the functions of the upregulated genes were enriched in 'cell cycle', 'ECM-receptor interaction', 'PI3K-Akt signaling pathway', 'p53 signaling pathway' and 'focal adhesion'. Extracellular matrix (ECM) rigidity may mediate the invasion of GBM multiforme cells through actomyosin contractility $(25,26)$. The PI3K-Akt signaling pathway serves an important role in glioma formation, through the suppression of cell death $(27,28)$. p53 is a tumor suppressor factor which initiates DNA repair, cell cycle arrest and apoptosis, and responds to numerous types of cancer therapy (29,30). Downregulated DEGs were enriched in 'morphine addiction', 'GABAergic synapse', 'retrograde endocannabinoid signaling', 'calcium signaling pathway' and 'glutamatergic synapse'. Calcium signaling has notable functions in numerous signaling processes involved in the proliferation and motility of GBM cells (31).

Analysis of the top two modules from the PPI network indicated that GBM was associated with the cell cycle and neuroactive ligand-receptor interaction. Pal et al (32) recently demonstrated that patients who have GBM in combination with a defective neuroactive ligand-receptor interaction pathway have a poor prognosis $(\mathrm{P}<0.0001)$. Therefore, monitoring these signaling pathways may help predict tumor occurrence and progression. The top 15 hub genes were identified from the network. Although these hub genes were all upregulated in GBM, VEGFA is the only gene which was significantly associated with the poor prognosis of patients with GBMs. GBMs are highly vascularized tumors, and VEGFA is highly expressed in the endothelial cells of blood vessels (33). Bevacizumab, a monoclonal antibody against VEGFA, improves the progression-free survival of patients with GBM, however it does not prolong the OS of patients compared with the historical control (34). Antiangiogenic treatment does not improve the OS of patients with GBM compared with standard cytotoxic treatment, thus, an in-depth understanding of the molecular mechanism underlying all of the hub genes of GBM, including CDK1, CDC20, AURKA, and BUB1, is required. CDK1, which is enriched in the module of Cluster 1, serves vital roles in regulating oncogenesis and cell cycle progression $(35,36)$. The overexpression of CDC20 is associated with temozolomide resistance in glioma cells (37). AURKA regulates the self-renewal and tumorigenicity of glioma-initiating cells through the stabilization of $\beta$-catenin (38).

Similar bioinformatics studies also used the expression profile of GSE4290 for their analysis. However, previous studies applied alternative bioinformatics methods to those used in the present study, and thus obtained different results. For example, two separate studies conducted bioinformatics analysis in accordance with pathological grading (e.g. astrocytoma, GBM and oligodendroglioma). One study reported that long-term potentiation and ECM-receptor interaction may have important roles in the occurrence and development of glioma, whereas the other study focused on the involvement 
of the Wnt and p53 signaling pathways in glioma $(39,40)$. Li et al (41) compared 81 GBM samples with 23 controls from GSE4290 and identified significant MAPK and cell cycle signaling pathways. Furthermore, they reported that a number of genes, including neuroblastoma RAS viral oncogene homolog, CDK2, fibroblast growth factor receptor 2, and cyclin D1, were associated with GBM (41). Wei et al (42) used a method similar to that used in the present study, in order to analyze 23 nontumor and 77 GBM (Grade 4) tumor samples of GSE4290. Through GO analysis, it was discovered that DEGs were enriched in 'synaptic transmission', 'regulation of vesicle-mediated transport' and 'ion-gated channel activity'. KEGG analysis results indicated that DEGs were enriched in 'neuroactive ligand-receptor interaction', 'calcium signaling pathway', 'p53 signaling pathway' and 'cell cycle'. The study also identified vital transcription factors, including tumor protein $\mathrm{p} 53$, specificity protein 1 , JUN proto-oncogene AP-1 transcription factor subunit, signal transducer and activator of transcription 3, and transcription factor PU.1 (42). In the present study, only GBM samples were included, and not astrocytoma or oligodendroglioma. Expression and survival analyses were also conducted using the novel GEPIA tool and the TCGA and GTEx databases. Therefore, the results of the present study expand on the current knowledge and understanding of the molecular mechanisms of GBM.

In conclusion, bioinformatics analysis identified hub genes and pathways that may have central roles in the occurrence, development and prognosis of GBM. VEGFA, CDK1, CDC20, AURKA and BUB1, the hub genes of GBM, may serve important roles in the diagnosis and treatment of GBM.

\section{Acknowledgements}

Not applicable.

\section{Funding}

No funding was received.

\section{Availability of data and materials}

The datasets analyzed during the current study are available in the GEO repository (http://www.ncbi.nlm.nih.gov/geo/).

\section{Author's contributions}

WL was in charge of study design. SY and KG were in charge of data analysis and article publication.

\section{Ethics approval and consent to participate}

Not applicable.

\section{Patient consent for publication}

Not applicable.

\section{Competing interests}

The authors declare that they have no competing interests.

\section{References}

1. Dolecek TA, Propp JM, Stroup NE and Kruchko C: CBTRUS statistical report: Primary brain and central nervous system tumors diagnosed in the United States in 2005-2009. Neuro Oncol 14 (Suppl 5): v1-v49, 2012.

2. Stupp R, Mason WP, van den Bent MJ, Weller M, Fisher B, Taphoorn MJ, Belanger K, Brandes AA, Marosi C, Bogdahn U, et al: Radiotherapy plus concomitant and adjuvant temozolomide for glioblastoma. N Engl J Med 352: 987-996, 2005.

3. Wen PY and Kesari S: Malignant gliomas in adults. N Engl J Med 359: 492-507, 2008.

4. Yeom SY,Nam DH and Park C: RRAD promotes EGFR-mediated STAT3 activation and induces temozolomide resistance of malignant glioblastoma. Mol Cancer Ther 13: 3049-3061, 2014.

5. Zhang Z, Lu J, Guo G, Yang Y, Dong S, Liu Y, Nan Y, Zhong Y, Yu K and Huang Q: IKBKE promotes glioblastoma progression by establishing the regulatory feedback loop of IKBKE/YAP1/miR-Let-7b/i. TumourBiol39: 1010428317705575, 2017.

6. Kaynar MY, Sanus GZ, Hnimoglu H, Kacira T, Kemerdere R, Atukeren P, Gumustas K, Canbaz B and Tanriverdi T: Expression of hypoxia inducible factor-1alpha in tumors of patients with glioblastoma multiforme and transitional meningioma. J Clin Neurosci 15: 1036-1042, 2008.

7. Qiu Z, Yuan W, Chen T, Zhou C, Liu C, Huang Y, Han D and Huang Q: HMGCR positively regulated the growth and migration of glioblastoma cells. Gene 576: 22-27, 2016.

8. Gao X, Mi Y, Ma Y and Jin W: LEF1 regulates glioblastoma cell proliferation, migration, invasion, and cancer stem-like cell self-renewal. Tumour Biol 35: 11505-11511, 2014.

9. Sun L, Hui AM, Su Q, Vortmeyer A, Kotliarov Y, Pastorino S, Passaniti A, Menon J, Walling J, Bailey R, et al: Neuronal and glioma-derived stem cell factor induces angiogenesis within the brain. Cancer Cell 9: 287-300, 2006.

10. Davis S and Meltzer PS: GEOquery: A bridge between the gene expression omnibus (GEO) and BioConductor. Bioinformatics 23: 1846-1847, 2007.

11. Ashburner M, Ball CA, Blake JA, Botstein D, Butler H, Cherry JM, Davis AP, Dolinski K, Dwight SS, Eppig JT, et al: Gene ontology: Tool for the unification of biology. The Gene Ontology Consortium. Nat Genet 25: 25-29, 2000.

12. Kanehisa M and Goto S: KEGG: Kyoto encyclopedia of genes and genomes. Nucleic Acids Res 28: 27-30, 2000.

13. Deng L, Xiong P, Luo Y, Bu X, Qian S and Zhong W: Bioinformatics analysis of the molecular mechanism of diffuse intrinsic pontine glioma. Oncol Lett 12: 2524-2530, 2016.

14. Ma K, Cheng Z, Sun L and Li H: Identification of potential therapeutic targets for gliomas by bioinformatics analysis. Oncol Lett 14: 5203-5210, 2017.

15. Sun C, Yuan Q, Wu D, Meng X and Wang B: Identification of core genes and outcome in gastric cancer using bioinformatics analysis. Oncotarget 8: 70271-70280, 2017.

16. Jiao X, Sherman BT, Huang da W, Stephens R, Baseler MW, Lane HC and Lempicki RA: DAVID-WS: A stateful web service to facilitate gene/protein list analysis. Bioinformatics 28 : 1805-1806, 2012.

17. Szklarczyk D, Franceschini A, Wyder S, Forslund K, Heller D, Huerta-Cepas J, Simonovic M, Roth A, Santos A, Tsafou KP, et al: STRING v10: Protein-protein interaction networks, integrated over the tree of life. Nucleic Acids Res 43 (Database Issue): D447-D452, 2015.

18. Tang Z, Li C, Kang B, Gao G, Li C and Zhang Z: GEPIA: A web server for cancer and normal gene expression profiling and interactive analyses. Nucleic Acids Res 45: W98-W102, 2017.

19. Behin A, Hoang-Xuan K, Carpentier AF and Delattre JY: Primary brain tumours in adults. Lancet 361: 323-331, 2003.

20. Fueyo J, Gomez-Manzano C, Alemany R, Lee PS, McDonnell TJ, Mitlianga P, Shi YX, Levin VA, Yung WK and Kyritsis AP: A mutant oncolytic adenovirus targeting the $\mathrm{Rb}$ pathway produces anti-glioma effect in vivo. Oncogene 19: 2-12, 2000.

21. Fueyo J, Gomez-Manzano C, Liu TJ and Yung WK: Delivery of cell cycle genes to block astrocytoma growth. J Neurooncol 51: 277-287, 2001.

22. Alexiou GA, Vartholomatos G, Goussia A, Batistatou A, Tsamis K, Voulgaris S and Kyritsis AP: Fast cell cycle analysis for intraoperative characterization of brain tumor margins and malignancy. J Clin Neurosci 22: 129-132, 2015. 
23. Yu LJ, Wall BA, Wangari-Talbot J and Chen S: Metabotropic glutamate receptors in cancer. Neuropharmacology 115: 193-202, 2017.

24. Wang Y, Jiang M, Yao Y and Cai Z: WWC3 inhibits glioma cell proliferation through suppressing the $\mathrm{Wnt} / \beta$-catenin signaling pathway. DNA Cell Biol 37: 31-37, 2018.

25. Ulrich TA, de Juan Pardo EM and Kumar S: The mechanical rigidity of the extracellular matrix regulates the structure, motility, and proliferation of glioma cells. Cancer Res 69: 4167-4174, 2009.

26. Bellail AC, Hunter SB, Brat DJ, Tan C and Van Meir EG: Microregional extracellular matrix heterogeneity in brain modulates glioma cell invasion. Int J Biochem Cell Biol 36: 1046-1069, 2004.

27. Cantrell DA: Phosphoinositide 3-kinase signalling pathways. J Cell Sci 114: 1439-1445, 2001.

28. Ashcroft M, Ludwig RL, Woods DB, Copeland TD, Weber HO, Macrae EJ and Vousden KH: Phosphorylation of HDM2 by Akt. Oncogene 21: 1955-1962, 2002.

29. Vassilev LT, Vu BT, Graves B, Carvajal D, Podlaski F, Filipovic Z Kong N, Kammlott U, Lukacs C, Klein C, et al: In vivo activation of the p53 pathway by small-molecule antagonists of MDM2. Science 303: 844-848, 2004

30. Bond GL, Hu W, Bond EE, Robins H, Lutzker SG, Arva NC, Bargonetti J, Bartel F, Taubert H, Wuerl P, et al: A single nucleotide polymorphism in the MDM2 promoter attenuates the p53 tumor suppressor pathway and accelerates tumor formation in humans. Cell 119: 591-602, 2004.

31. Kang SS, Han KS, Ku BM,Lee YK, Hong J, Shin HY, Almonte AG, Woo DH, Brat DJ, Hwang EM, et al: Caffeine-mediated inhibition of calcium release channel inositol 1,4,5-trisphosphate receptor subtype 3 blocks glioblastoma invasion and extends survival. Cancer Res 70: 1173-1183, 2010.

32. Pal J, Patil V, Kumar A, Kaur K, Sarkar C and Somasundaram K: Loss-of-function mutations in Calcitonin receptor (CALCR) identify highly aggressive glioblastoma with poor outcome. Clin Cancer Res 24: 1448-1458, 2018.

33. Wong ML, Prawira A, Kaye AH and Hovens CM: Tumour angiogenesis: Its mechanism and therapeutic implications in malignant gliomas. J Clin Neurosci 16: 1119-1130, 2009.
34. Yang SB, Gao KD, Jiang T, Cheng SJ and Li WB: Bevacizumab combined with chemotherapy for glioblastoma: A meta-analysis of randomized controlled trials. Oncotarget 8: 57337-57344, 2017.

35. Paruthiyil S, Cvoro A, Tagliaferri M, Cohen I, Shtivelman E and Leitman DC: Estrogen receptor $\beta$ causes a G2 cell cycle arrest by inhibiting CDK1 activity through the regulation of cyclin B1, GADD45A, and BTG2. Breast Cancer Res Treat 129: 777-784, 2011.

36. Fan X and Chen JJ: Role of Cdk1 in DNA damage-induced G1 checkpoint abrogation by the human papillomavirus E7 oncogene. Cell Cycle 13: 3249-3259, 2014.

37. Wang J, Zhou F, Li Y, Li Q, Wu Z, Yu L, Yuan F, Liu J, Tian Y, Cao Y, et al: Cdc20 overexpression is involved in temozolomide-resistant glioma cells with epithelial-mesenchymal transition. Cell Cycle 16: 2355-2365, 2017.

38. Xia Z, Wei P, Zhang H, Ding Z, Yang L, Huang Z and Zhang N: AURKA governs self-renewal capacity in glioma-initiating cells via stabilization/activation of $\beta$-catenin/Wnt signaling. Mol Cancer Res 11: 1101-1111, 2013.

39. Wang R, Wei J, Li Z, Tian Y and Du C: Bioinformatical analysis of gene expression signatures of different glioma subtypes. Oncol Lett 15: 2807-2814, 2018

40. Hu G, Wei B, Wang L, Wang L, Kong D, Jin Y and Sun Z: Analysis of gene expression profiles associated with glioma progression. Mol Med Rep 12: 1884-1890, 2015.

41. Li W, Li K, Zhao L and Zou H: Bioinformatics analysis reveals disturbance mechanism of MAPK signaling pathway and cell cycle in Glioblastoma multiforme. Gene 547: 346-350, 2014.

42. Wei B, Wang L, Du C, Hu G, Wang L, Jin Y and Kong D Identification of differentially expressed genes regulated by transcription factors in glioblastomas by bioinformatics analysis. Mol Med Report 11: 2548-2554, 2015.

cc (i) This work is licensed under a Creative Commons Attribution 4.0 International (CC BY 4.0) License. 